\title{
Evaluation and Treatment of Erectile Dysfunction
}

\author{
Maarten Albersen, $\mathrm{MD}^{\mathrm{a}}$, Kuwong B. Mwamukonda, $\mathrm{MD}^{\mathrm{b}}$, \\ Alan W. Shindel, $\mathbf{M D}^{\mathrm{C}}$, Tom F. Lue, $\mathrm{MD}^{\mathrm{d}, *}$
}

\section{KEYWORDS}

- Erectile dysfunction - Phosphodiesterase 5 inhibitors

- Apomorphine SL • Intracavernous injection • Prostaglandin

- Evaluation • Assessment $\bullet$ Treatment

Erectile dysfunction (ED) was defined by the National Institutes of Health Consensus Development Panel on Impotence as the persistent inability to attain and maintain an erection sufficient for sexual intercourse. ${ }^{1} \mathrm{ED}$ is the most thoroughly studied sexual dysfunction in men and the most common sexual complaint of men presenting to their health care providers. ${ }^{2}$ Various large-scale studies (both cross-sectional and longitudinal) have indicated that the worldwide prevalence of ED is between $10 \%$ and $20 \%$. ED is strongly correlated with aging, with a steep incline in prevalence rates, from $6.5 \%$ in men aged 20 to 39 years to $77.5 \%$ in those aged 75 years and older. ${ }^{3}$ ED is also associated with various comorbidities, including psychological factors, cardiovascular diseases, diabetes mellitus, and metabolic syndrome, and with smoking. ${ }^{4}$ latrogenic ED is not uncommon and can be the result of pelvic surgical procedures or the use of various medications. ${ }^{4}$

Although ED is not a direct threat to physical health, it can have dramatic effects on personal sense of well-being and has a significant impact on the quality of life of

Conflicts of interest: Dr Maarten Albersen has received an unrestricted educational grant from Bayer Healthcare Belgium and is a scholar of the European Society for Surgical Oncology, Belgische Vereniging voor Urologie, and the Federico Foundation.

Dr Kuwong B. Mwamukonda has no conflicts of interest to disclose.

Dr Alan W. Shindel has received a research grant from American Medical Systems, and is an informal consultant for Boehringer Ingelheim.

Dr Tom F. Lue is a consultant of Pfizer, Eli Lilly \& Co, Bayer, Medtronic, and Auxilium, and is a board member of Genix. He has received a research grant from American Medical Systems.

a Laboratory of Experimental Urology, Department of Urology, University Hospitals Leuven, Herestraat 49, Leuven 3000, Belgium

b SAUSHEC Urology Program, Brooke Army Medical Center, 3851 Roger Brooke Drive Fort Sam, Houston, TX 78234-6200, USA

c UC Davis Department of Urology, Lawrence J. Ellison Ambulatory Care Center, 4860 Y Street, Suite 2200, Sacramento, CA 95817, USA

d Department of Urology, University of California at San Francisco, 400 Parnassus Avenue, Campus Box 0738, San Francisco, CA 94143-0738, USA

* Corresponding author.

E-mail address: Tlue@urology.ucsf.edu 
patients and their sexual partners. ED is also an independent predictor of cardiovascular morbidity and mortality. ${ }^{5,6}$ For these reasons, ED merits consideration from the primary care physician as an important health concern and as a potential sentinel event for serious health problems. This article discusses the physiology and pathophysiology of erectile function in men, how the primary care physician may address the clinical problem of ED in practice, and when specialty referral is indicated.

\section{PHYSIOLOGY AND PATHOPHYSIOLOGY OF ED}

Penile erection is often described as a neurovascular process that is controlled by hormones. This article briefly describes the physiologic mechanisms of penile erection. A more elaborate discussion of the mechanisms of penile erection can be found elsewhere. ${ }^{4,7,8}$

\section{Physiology of Penile Erection}

During sexual stimulation, the hypothalamus is exposed to input from various neurotransmitters; dopamine seems to be the primary erectogenic central nervous system neurotransmitter. Dopamine-containing nerve endings impinge on oxytocinergic cell bodies contained in pathways descending from the hypothalamus to the brain stem and spinal autonomic centers and produce a "psychogenically mediated" erection. ${ }^{8} \mathrm{~A}$ "tactile-mediated" erectogenic stimulus may result from direct stimulation of the penis. This stimulus occurs via sensory neurons that synapse in the sacral spinal cord.

In either case, the cavernous nerves, which arise from the pelvic plexus, are directly responsible for conducting impulses that generate penile erection. These nerves run alongside the posterolateral side of the prostatic capsule and perforate the urogenital diaphragm to enter the cavernous bodies at the level of the crura. Cavernous nerve activation leads to the release of the gaseous neurotransmitter nitric oxide (NO) from the nonadrenergic, noncholinergic nerve terminals in the corpus cavernosum. Additional $\mathrm{NO}$ is released from the endothelium in response to shear stress and the release of acetylcholine from parasympathetic endothelial nerve endings. In both cases, NO is synthesized by the enzyme nitric oxide synthase (NOS), which converts oxygen and l-arginine to $\mathrm{NO}$ and citrulline in the cavernous nerve terminals ( $\mathrm{nNOS}$ ) and in the endothelium (eNOS). ${ }^{4,7,8}$

NO passively diffuses into smooth muscle cells in the arterial wall and the trabeculae of the corpus cavernosum, where it binds to and activates soluble guanylate cyclase (GC). GC then catalyzes the breakdown of guanosine triphosphate into 3'5'-cyclic guanosine monophosphate (cGMP). cGMP acts as a downstream messenger and initiates a chain of reactions ultimately resulting in a decrease of intracellular calcium and relaxation of the smooth muscle (Fig. 1). Although this pathway is functionally the most important means to produce smooth muscle relaxation, it is supported by the synergistic actions of other pathways using 3'5'-cyclic adenosine monophosphate (CAMP) as a second messenger.

cAMP and cGMP are hydrolyzed by a class of enzymes called phosphodiesterases, of which subtype 5 (PDE5), a cGMP-specific phosphodiesterase, seems to be the dominant active isoform in penile tissue. Vasodilation and relaxation of trabecular smooth muscle allow rapid blood flow into the cavernosal sinusoids and the development of an erection, which is maintained by the compression of subtunical venules against the tunica albuginea, and reinforced by contraction of the voluntary ischiocavernosus muscle during the rigid erection phase. These pro-erectogenic parasympathetic pathways are counterbalanced by several sympathetically mediated 
mechanisms that maintain penile flaccidity. These mechanisms are less relevant for this review and are discussed in detail elsewhere. ${ }^{4,9}$

\section{Pathophysiology of ED}

Penile erection requires neural transmission of pro-erectile impulses, an intact arterial blood supply, and functional erectile tissue in the corpus cavernosum. ED can develop from a defect in one of these tissues or, more commonly, from a defect in a combination of tissues. ED is classified as vasculogenic, neurogenic, hormonal, anatomic/structural, drug-induced, or psychogenic. ${ }^{4,10}$ Table 1 summarizes the most prevalent causes of ED.

ED is strongly associated with various comorbidities, including cardiovascular diseases, diabetes mellitus, metabolic syndrome, and late-onset hypogonadism, and with smoking. ${ }^{8}$ Aging is another major risk factor for ED. ${ }^{11}$ These comorbid conditions and their relationship with ED will be discussed more in detail in the article by Berookhim and Bar-Chama elsewhere in this issue.

\section{ASSESSMENT OF ED Medical History}

ED is often multifactorial in origin and is therefore best managed with a holistic approach that includes lifestyle modification, pharmacologic treatment, and attention to the relationship between partners. It is of particular importance to encourage open and honest communication between the patient and partner. All of these approaches are best facilitated in the context of a trusting patient/provider relationship, and therefore providers must establish rapport with patients when addressing issues of sexuality. ${ }^{10,11}$

The initial evaluation of ED should include a complete medical, psychosocial, and sexual history. A thorough medical assessment is mandatory in evaluating erectile complaints, particularly in older men and patients at intermediate and high risk for cardiovascular disease. ${ }^{10}$ These patients should undergo cardiovascular assessment before continuing sexual activity and before beginning therapy for ED (Fig. 2). ${ }^{12}$ Signs and symptoms of possible underlying conditions should be assessed, such as depression, diabetes, late-onset hypogonadism, metabolic syndrome, and medication/surgically induced causes of ED. Information about the association between ED and tobacco use can be an important tool in helping patients decide to quit use of tobacco products. ${ }^{10,13} \mathrm{~A}$ thorough review of current medications may reveal agents that are known to cause or exacerbate ED (eg, antidepressants, antiandrogens, thiazides, and $\beta$-blockers). It is also important to assess for use of nitrate-donors, which are absolute contraindications for therapy with PDE5 inhibitors, and $\alpha$-blockers that require an interval between use of the two drugs. ${ }^{10,14}$ The goal of the history should be to not only understand the specific erectile condition but also identify possible underlying and reversible or treatable disorders.

\section{Sexual History}

The use of validated questionnaires, such as the International Index of Erectile Function, can be useful as an "ice-breaker" to initiate the conversation about ED. Numeric scores from instruments such as this may also be helpful in assessing the severity of $\mathrm{ED}$, screening for other sexual dysfunctions, and evaluating treatment outcome. ${ }^{15,16}$ These metrics ,however, should not be regarded as a replacement for direct assessment of sexual history. ${ }^{13}$ An adequate sexual history should include information about current sexual relationships, the emotional status of the patient and the partner, and the exact nature of the couple's sexual concerns. ${ }^{10}$ Issues of sexual orientation and gender identity should also be noted. ${ }^{13}$ Descriptive measures such as rigidity 


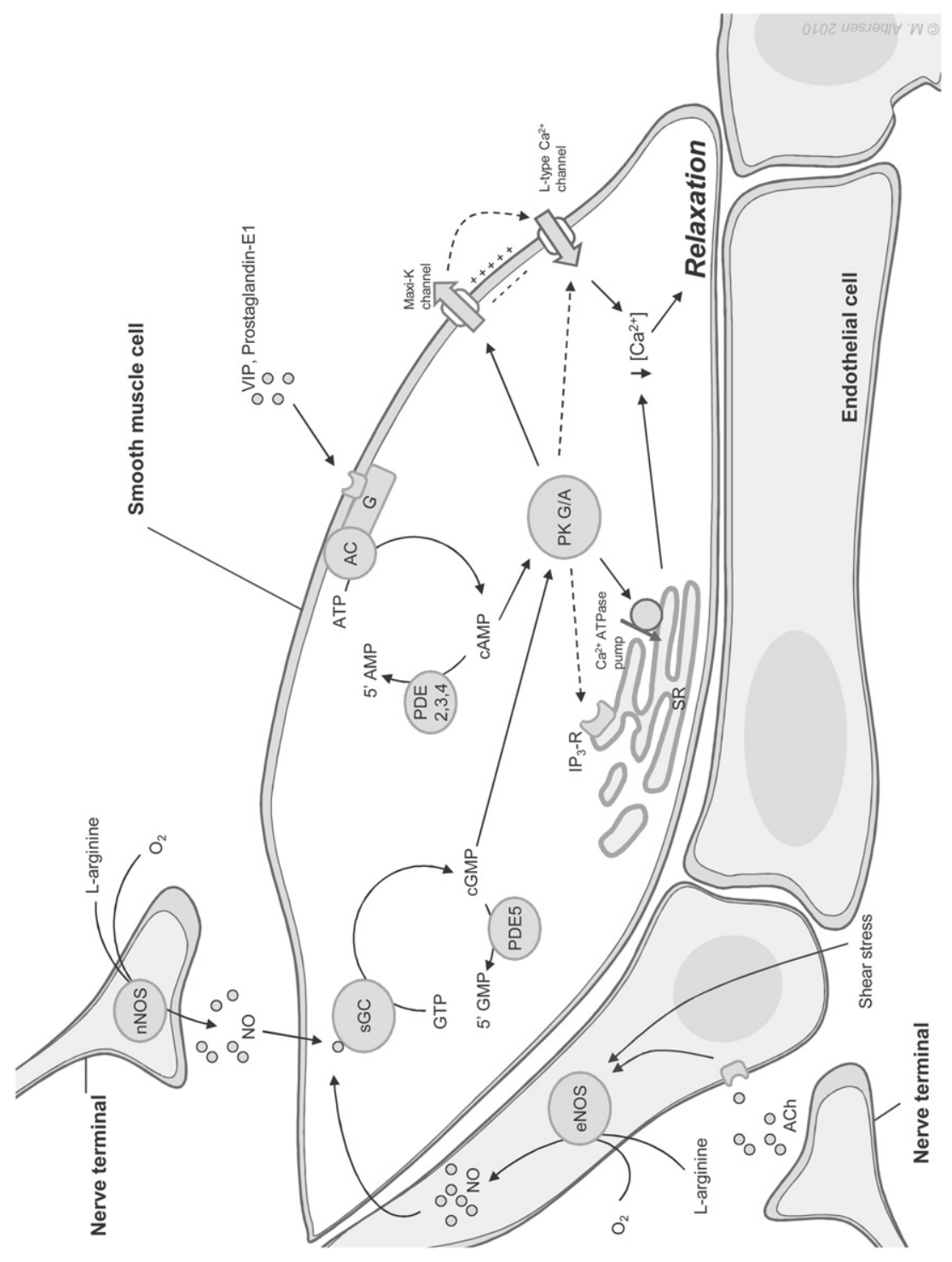


and duration of nocturnal erections, erections during masturbation, and erections after sexual arousal should be discussed, because they can give clues about the cause of ED. The onset of the problem and any situational factors that ameliorate or exacerbate ED should be determined. Problems with arousal, ejaculation, and difficulty reaching orgasm should be discussed because these may be signs of concomitant sexual dysfunctions requiring specialist assessment. ${ }^{10}$ The impact of ED and other sexual dysfunctions on general well-being and sexual satisfaction, and issues of partner interest in and satisfaction with sex should be discussed.

\section{Physical Examination}

A complete external genital examination should be performed to detect anatomic or structural deformities of the penis, such as Peyronie plaques. Assessment of testicular size is important because small testes and regression of secondary sex characteristics may indicate hypogonadism. ${ }^{10,11,13}$ Physical examination should also include a general screening for risk factors and comorbidities that are associated with ED, such as cardiovascular disease, neurologic disease, and obesity. Blood pressure and heart rate should be measured if they were not assessed in the previous 3 to 6 months. ${ }^{10}$

\section{Laboratory Testing}

Recommended laboratory tests include a complete blood cell count and measurements of fasting serum glucose, a lipid profile, and free and total testosterone, particularly in patients with signs of hypogonadism. ${ }^{10,11,13}$ Additional hormonal testing is only required when low testosterone levels are detected. Baseline prostate-specific antigen screening is advised in patients older than 40 years or when ED is accompanied by lower urinary tract symptoms. ${ }^{17}$

\section{Specific Diagnostic Testing}

Radiologic testing, nocturnal penile rigidity testing, vascular and neurologic functional testing, and penile Doppler ultrasound are available for further diagnostic workup of ED. These tests are not routinely indicated in the primary care setting but may be ordered by urologists or sexual medicine specialists in certain cases. ${ }^{4,10}$

Fig. 1. Molecular mechanisms of penile smooth muscle relaxation. Nitric oxide (NO) is released from nerve terminals in the corpus cavernosum in response to a neural stimulus and from the endothelium in response to the release of acetylcholine (Ach) and the shear stress elicited by increased blood flow in the corporeal sinusoids. NO binds to soluble guanylate cyclase (sGC) and thereby activates this enzyme, which catalyzes the breakdown of guanosine triphosphate (GTP) into cyclic guanosine monophosphate (cGMP). Other pathways, which are initiated by vasoactive intestinal polypeptide and prostaglandin E1, activate a $G$ protein (G)-coupled receptor, leading to activation of adenylate cyclase (AC), which catalyzes the breakdown of adenosine triphosphate (ATP) into cyclic adenosine monophosphate (CAMP). cGMP and CAMP exert analogous effects by activating protein kinase $G$ and $A$ (PK G/A), respectively, which modulate potassium and calcium channels in the cell membrane and the inositol triphosphate receptor $\left(I_{3}-R\right)$ and the calcium-ATPase pump in the membrane of the sarcoplasmic reticulum (SR). These events lead to a lowering of the cytosolic calcium concentration, which causes dissociation of calcium from calmodulin. Calmodulin then dissociates from myosin light chain kinase, thus inactivating it, leading to smooth muscle relaxation and ultimately penile tumescence. eNOS, endothelial nitric oxide synthase; NOS, neuronal nitric oxide synthase; PDE, phosphodiesterase; VIP, vasoactive intestinal polypeptide. (Adapted from Albersen M, Shindel AW, Mwamukonda KB, et al. The future is today: emerging drugs for the treatment of erectile dysfunction. Expert Opin Emerg Drugs 2010;15:467-80; with permission from Informa Healthcare.) 


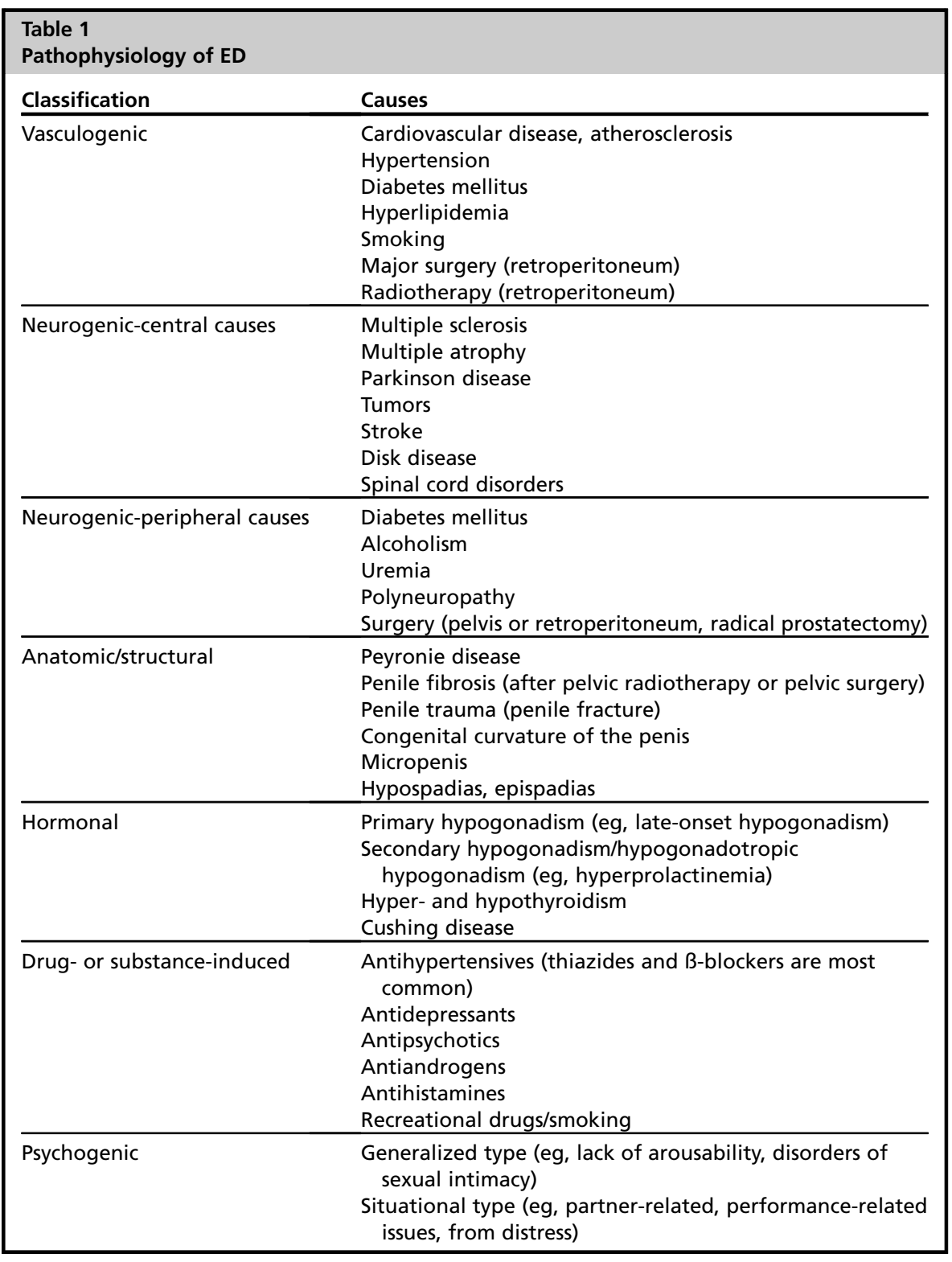

Adapted from Wespes E , Amar E, Eardley I, et al. EAU Guidelines on Male Sexual Dysfunction: erectile dysfunction and premature ejaculation. (c) European Association of Urology 2009; with permission.

\section{When to Refer}

The decision of when to refer a patient for management of ED must be made on a case-by-case basis and is determined in large part by the interest and comfort a given primary care provider has in managing ED. Cases that should prompt immediate referral include patients with lifelong $\mathrm{ED}$, a history of pelvic, urethral or perineal 


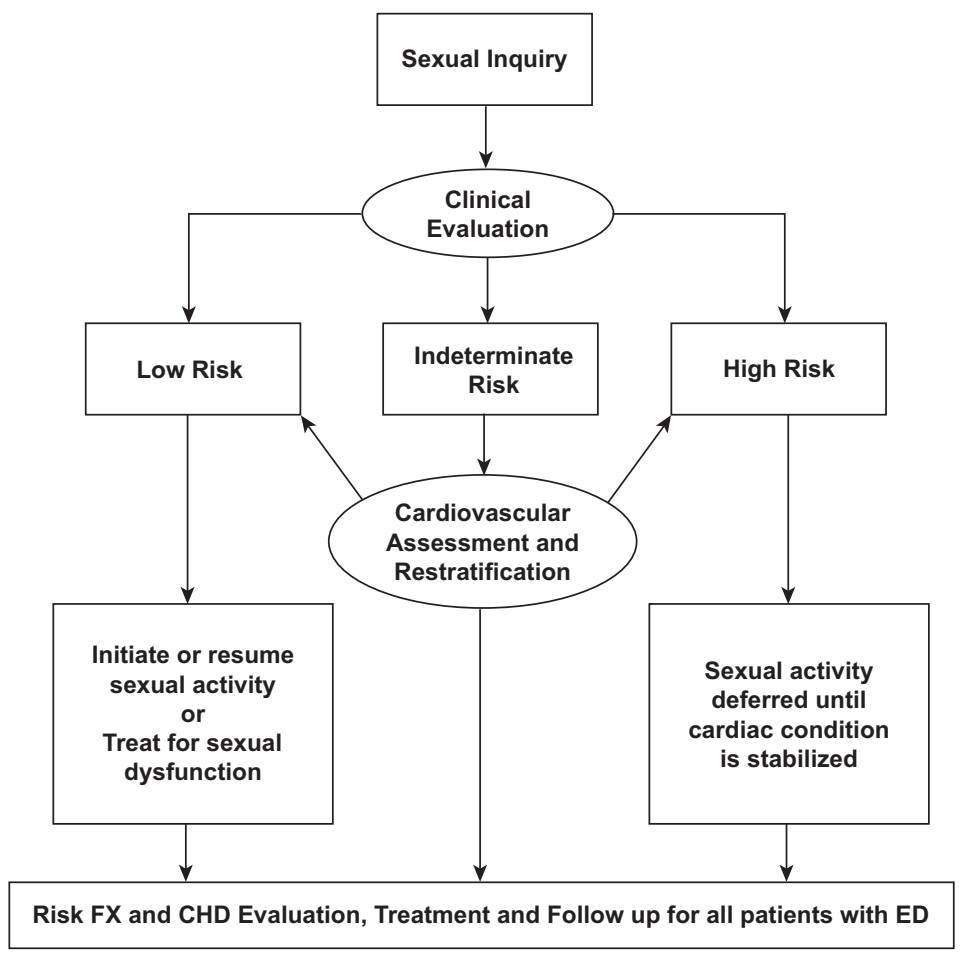

Fig. 2. Sexual dysfunction and cardiac risk (the second Princeton Consensus Conference). CHD, coronary heart disease; Fx, risk factors. (Reprinted from Kostis JB, Jackson G, Rosen $\mathrm{R}$, et al. Sexual dysfunction and cardiac risk (the Second Princeton Consensus Conference). Am J Cardiol 2005;96(2):313-21. (C) 2005; with permission from Elsevier.)

trauma, penile deformities, endocrine disorders detected during standard laboratory testing, and complex or severe psychiatric or psychosexual disorders. ${ }^{10}$

\section{TREATMENT OF ED IN THE PRIMARY CARE SETTING General Considerations Before Initiating Pharmacologic Treatment}

Both the patient's and partner's understanding of ED and results of the diagnostic tests should be reviewed before treatment is initiated, so that a rational selection of treatment options and expectations can be provided. Current pharmacologic treatments for ED do not cure ED but can generally be relied on to greatly improve erectile function. Setting realistic treatment goals and granting permission and legitimacy for engaging in alternative means of sexual intimacy that do not rely on penetrative sexual intercourse should be a goal of therapy. Few older patients with ED will be able to regain full potency, but most should be able to experience restoration of satisfying sexual encounters.

Underlying reversible conditions (obesity, medical comorbidities, relationship issues) should be addressed and treated before or simultaneously with initiating specific ED-directed treatment. ${ }^{18}$ Lifestyle changes, such as exercise and smoking cessation, should be suggested where applicable. Referral to exercise physiologists, nutritionists, or personal trainers may be of some benefit in these situations. 


\section{Oral Pharmacotherapy}

Although many treatment modalities are available for ED, this article mainly focuses on oral pharmacotherapy, which will efficiently treat most patients experiencing ED. When oral therapies fail, referral to a specialist should be considered except when the primary care provider has a particular interest and comfort in dealing with sexuality issues.

\section{PDE5-specific inhibitors}

PDE5-specific inhibitors (PDE5Is) are nonhydrolyzable analogs of cGMP and exert their beneficial effects on smooth muscle relaxation through competitively binding to the catalytic site of PDE5. Through slowing the degradation of CGMP, these drugs produce an intracellular accumulation of cGMP in smooth muscle cells in the arteries and trabeculae of the corpus cavernosum (see Fig. 1), resulting in relaxation of the smooth muscle, increased arterial blood flow, and penile tumescence. ${ }^{19}$

\section{Treatment of ED with PDE5I}

In current treatment guidelines, PDE5Is are recommended as the preferred pharmacotherapy for ED. ${ }^{10}$ Numerous trials have established on-demand efficacy rates of $60 \%$ to $70 \%$ in the general population, and postmarketing data confirms excellent safety profiles of the three compounds currently available in the United States (sildenafil, vardenafil, and tadalafil). ${ }^{19-22}$ Although a large crossover study showed an overall equivalence in the subjective perception of treatment benefits among all PDE5Is, the three currently available drugs differ from each other in time to onset of action and duration of action (sildenafil and vardenafil up to 5 hours and tadalafil up to $24-36$ hours). ${ }^{23}$ The choice of appropriate drug is based on patient and partner preference guided by physician advice. ${ }^{20}$

Before initiation of treatment, patients should be informed that sexual stimulation is essential for the efficacy of the drugs. Although some men may experience limited efficacy after a first trial, these patients should be informed that results generally improve with repeated dosing, and a minimum of six attempts should be made before treatment is considered a failure. The recommended starting doses are $50 \mathrm{mg}$ for sildenafil and $10 \mathrm{mg}$ for vardenafil and tadalafil. The unique pharmacokinetic properties of tadalafil have led to the approval of this drug as a daily treatment for ED at 2.5- and 5-mg doses; this regimen may be best for patients who have frequent intercourse or those who desire to separate the act of taking the drug from sexual interactions. The lowest therapeutic doses ( $25 \mathrm{mg}$ of sildenafil and $5 \mathrm{mg}$ of vardenafil and tadalafil on-demand) should be used when liver or kidney failure is present or when the patient uses medication that inhibits the CYP34A pathway. Examples of these drugs are ketoconazole, itraconazole, erythromycin, clarithromycin, and HIV protease inhibitors. ${ }^{10}$ However, patients taking medications that potentiate the CYP34A pathway, such as rifampin, phenobarbital, phenytoin, and carbamazepine, may need higher doses for efficacy. ${ }^{10}$

\section{Safety profile}

Postmarketing surveillance has not shown increased myocardial infarction rates in patients using PDE5ls compared with age-matched controls. ${ }^{21}$ However, certain heart-related precautions must be considered in men taking PDE5Is. PDE5Is are relatively contraindicated in patients with unstable angina pectoris, recent myocardial infarction, certain arrhythmias, and poorly controlled hypertension. These patients should undergo cardiovascular examination and treatment for their heart-related condition before initiating ED treatment. ${ }^{10}$ Furthermore, patients treated with nitrates or nitrate-donors should not take PDE5Is, and, use of PDE5Is with certain $\alpha$-blockers 
may result in postural hypotension. ${ }^{10}$ Patients taking $\alpha$-blockers for prostate symptoms or blood pressure control should take PDE5IS with at least a 4-hour window between dosing because of a theoretical risk of orthostatic hypotension, although some evidence suggests that the risk is low in patients on long-term $\alpha$-blocker therapy. ${ }^{14}$ Patients with prolongation of the QTc interval should not be treated with vardenafil. ${ }^{22}$

\section{Adverse events}

The most common adverse events from PDE5Is include headache, facial and ocular hyperemia, nasal congestion, myalgia, dyspepsia, and back pain. These side effects are attributable to specific inhibition of PDE5 and subsequent vasodilatation in tissues other than the penis. ${ }^{7,21}$ Congestion and flushing are more common with sildenafil relative to the other PDE5Is, whereas myalgias and dyspepsia are more strongly associated with tadalafil and vardenafil, respectively. Other less-common adverse events involve visual disturbances, most often attributable to the inhibition of PDE6 in the cones of the retina. ${ }^{7}$ Adverse events account for approximately $25 \%$ of patients who discontinue PDE5I use, whereas the most common reason for discontinuation of PDE5ls is lack of efficacy. ${ }^{24}$ More serious adverse events are rare and include seizures, nonarteritic ischemic optic neuritis, and acute hearing loss, although the exact role of PDE5Is in these conditions is debatable and reports are anecdotal. ${ }^{21,25}$ Patients can generally be reassured that the risk of serious long-term adverse events is low in appropriately selected candidates for PDE5I therapy.

\section{Nonresponders}

Of the patients who do not experience an initial response to PDE5I, between $30 \%$ and $50 \%$ may be converted to responders through re-education on proper dosing technique and through dose-escalation. Patients who discontinue tadalafil because of side effects may benefit from the daily-dosing option (2.5 or $5 \mathrm{mg} / \mathrm{d}) .{ }^{26-28}$ Furthermore, addition of exogenous testosterone supplementation may enhance PDE5I therapy in individuals in whom hypogonadism is confirmed. ${ }^{29}$ Because the efficacy of PDE5I depends on the integrity of the NO pathway in producing cGMP, patients in whom this pathway is disturbed will benefit far less from PDE5I treatment compared with the general population. Disease states that diminish NO availability include denervation of the erectile tissue after radical prostatectomy; severe diabetes; and downregulation of NOS expression, as may be seen in atherosclerosis, metabolic syndrome, aging, and hypogonadism. ${ }^{7}$ These difficult-to-treat patients benefit from referral to a sexual medicine specialist or urologist for second-line treatment.

\section{Alternative Oral Pharmacotherapy}

\section{Apomorphine SL}

Apomorphine is a centrally acting nonselective dopamine agonist that exhibits D2-like effects. It acts by binding to dopamine receptors in the hypothalamus and enhances naturally occurring pro-erectile signals. ${ }^{30}$ It is available in 2- or 3-mg doses but has not been approved in the United States for the treatment of ED. ${ }^{19}$ It is rapidly absorbed through the sublingual route of administration and results in the development of an erection within 20 minutes in more than two-thirds of patients. ${ }^{30}$ Apomorphine has lower efficacy and satisfaction rates than PDE5Is, and is most effective in patients with mild to moderate ED. ${ }^{10}$ It also is a valid alternative to PDE5Is for patients in whom psychogenic ED is suspected and in those who have contraindications to PDE5Is, such as those taking nitrates. The most common adverse events are caused by nonspecific binding to other subtypes of the dopamine receptor and include nausea, headache, and dizziness. ${ }^{30}$ These effects occur with relative high frequency, and have limited the usefulness of this drug. 


\section{Yohimbine}

Yohimbine is a natural peripherally and centrally acting $\alpha$-blocker. However, little evidence shows its efficacy in treating ED, and therefore it is not currently recommended in most guidelines for management of ED. It may have a role in patients who prefer natural products but should not be considered a recommended mainstream medical therapy. ${ }^{10}$

\section{Vacuum Constriction Devices}

The vacuum constriction device (VCD) creates negative pressure around the penis, thereby initiating passive engorgement of the sinusoidal spaces and creating an erection. Maintenance of erection is facilitated by application of a rubber cuff worn around the base of the penis. Although effective in up to $90 \%$ of patients, the use of a vacuum device might be perceived as disruptive, especially by younger men. ${ }^{10}$ Local side effects are relatively minor and include bruising, some discomfort, and ejaculatory obstruction. It is advised to limit the use of the constriction band to 30 minutes to avoid skin necrosis. Contraindications to VCD use include bleeding disorders and the use of anticoagulants. ${ }^{10}$

\section{Second-Line Treatment}

\section{Intracavernous and intraurethral therapy}

Before the advent of PDE5I,s intracavernous and intraurethral administration were the only nonsurgical treatment options for ED. The most commonly used substance, and currently the only one approved by the U.S. Food and Drug Administration as a treatment of ED, is prostaglandin E1 (PGE1). PGE1 activates adenylate cyclase and thereby raises intracellular CAMP, with effects analogous to those of cGMP (see Fig. 1). These effects are independent of the NO-cGMP pathway, making this treatment an excellent option for patients who do not experience response to PDE5I therapy. ${ }^{31}$ Intracavernous PGE1 therapy has an overall satisfaction rate of approximately $80 \% .{ }^{32}$

Phentolamine, papaverine, and vasoactive intestinal peptide are also available for intracavernous injection, although their role is limited to combination therapy (commonly referred to as bimix or trimix). ${ }^{11}$ PGE1 is also available for intraurethral administration (medicated urethral system for erection [MUSE]).

Adverse events from these therapies include priapism, variable degrees of pain with injection in approximately half of patients, and penile fibrosis after long-term use. ${ }^{31}$ Patients are advised to consult their physician if they experience prolonged penile pain or an erection lasting up to or more than 4 hours, because aspiration of cavernous blood may be necessary for penile decompression. ${ }^{10}$ Relative contraindications to injection therapy include a history of priapism or bleeding disorders. Before initiation of therapy, patients follow a short in-office training program. MUSE has many side effects in common with intracavernous PGE1, although it is less likely to cause priapism and may have marginal efficacy in many cases. MUSE has also been associated with hypotension, syncope, urethral burning or pain, and vaginal irritation in the partner. ${ }^{31}$

\section{Third-Line Therapy (Surgery)}

Implantation of a penile prosthesis, which can be either inflatable or malleable, is indicated for men in whom pharmacologic therapy is not effective. Implantation of a penile prosthesis has satisfaction rates of $70 \%$ to $90 \%$, but patients should be aware of the definitive and irreversible nature of this surgery. ${ }^{10}$ Adverse events include mechanical failure after several years of use (50\% after a 10 -year interval), infection (1\%-3\%), and, rarely, erosion. ${ }^{10,11}$

Other surgical options available for ED include penile revascularization and venous ligation. Outcomes of these surgeries in the general population of patients with ED are 
poor. These surgeries should be reserved for a select group of primarily young patients and should be performed in specialized centers only. ${ }^{10,11}$

\section{SUMMARY}

ED is a prevalent and important disease that has been associated with various comorbidities. The evaluation of patients with ED should include a general health assessment followed by a discussion of reversible factors and lifestyle changes that might help preserve erectile capacity. Numerous effective treatment options are currently available. A frank discussion about use and side effects of these therapies is required to optimize success. Although oral pharmacologic treatments can be initiated and monitored by the primary care physician, patients who do not experience response to these treatments may be best served by referral to a sexual medicine specialist for further assessment and consideration of other treatment options.

\section{REFERENCES}

1. NIH Consensus Conference. Impotence. NIH consensus development panel on impotence. JAMA 1993;270(1):83-90.

2. Uckert S, Mayer ME, Stief CG, et al. The future of the oral pharmacotherapy of male erectile dysfunction: things to come. Expert Opin Emerg Drugs 2007; 12(2):219-28.

3. Saigal CS, Wessells H, Pace J, et al. Predictors and prevalence of erectile dysfunction in a racially diverse population. Arch Intern Med 2006;166(2):207-12.

4. Lue TF. Erectile dysfunction. N Engl J Med 2000;342(24):1802-13.

5. Araujo AB, Travison TG, Ganz P, et al. Erectile dysfunction and mortality. J Sex Med 2009;6(9):2445-54.

6. Guo W, Liao C, Zou Y, et al. Erectile dysfunction and risk of clinical cardiovascular events: a meta-analysis of seven cohort studies. J Sex Med 2010. [Epub ahead of print].

7. Albersen M, Shindel AW, Mwamukonda KB, et al. The future is today: emerging drugs for the treatment of erectile dysfunction. Expert Opin Emerg Drugs 2010; 15(3):467-80.

8. Gratzke C, Angulo J, Chitaley K, et al. Anatomy, physiology, and pathophysiology of erectile dysfunction. J Sex Med 2010;7(1 Pt 2):445-75.

9. Lin CS, Xin ZC, Wang Z, et al. Molecular Yin and Yang of erectile function and dysfunction. Asian J Androl 2008;10(3):433-40.

10. Hatzimouratidis K, Amar E, Eardley I, et al. Guidelines on male sexual dysfunction: erectile dysfunction and premature ejaculation. Eur Urol 2010. [Epub ahead of print].

11. Albersen M, Shindel AW, Lue TF. Sexual dysfunction in the older man. Rev Clin Gerontol 2009;19:b1-8.

12. Kostis JB, Jackson $G$, Rosen $R$, et al. Sexual dysfunction and cardiac risk (the second Princeton Consensus Conference). Am J Cardiol 2005;96(2):313-21.

13. Hackett G, Kell P, Ralph D, et al. British Society for Sexual Medicine guidelines on the management of erectile dysfunction. J Sex Med 2008;5(8):1841-65.

14. Kloner RA. Pharmacology and drug interaction effects of the phosphodiesterase 5 inhibitors: focus on alpha-blocker interactions. Am J Cardiol 2005;96(12B): 42M-6M.

15. Rosen RC, Cappelleri JC, Gendrano N III. The international index of erectile function (IIEF): a state-of-the-science review. Int J Impot Res 2002;14(4):226-44. 
16. Rosen RC, Cappelleri JC, Smith MD, et al. Development and evaluation of an abridged, 5-item version of the International index of erectile function (IIEF-5) as a diagnostic tool for erectile dysfunction. Int J Impot Res 1999;11(6):319-26.

17. Greene KL, Albertsen PC, Babaian RJ, et al. Prostate specific antigen best practice statement: 2009 update. J Urol 2009;182(5):2232-41.

18. Esposito K, Giugliano F, Di Palo C, et al. Effect of lifestyle changes on erectile dysfunction in obese men: a randomized controlled trial. JAMA 2004;291(24): 2978-84.

19. Eardley I, Donatucci C, Corbin J, et al. Pharmacotherapy for erectile dysfunction. J Sex Med 2010;7(1 Pt 2):524-40.

20. Hatzimouratidis K, Hatzichristou DG. A comparative review of the options for treatment of erectile dysfunction: which treatment for which patient? Drugs 2005;65(12):1621-50.

21. Shindel AW. 2009 update on phosphodiesterase type 5 inhibitor therapy part 2: updates on optimal utilization for sexual concerns and rare toxicities in this class. J Sex Med 2009;6(9):2352-64.

22. Carson CC III. Cardiac safety in clinical trials of phosphodiesterase 5 inhibitors. Am J Cardiol 2005;96(12B):37M-41M.

23. Jannini EA, Isidori AM, Gravina GL, et al. The ENDOTRIAL study: a spontaneous, open-label, randomized, multicenter, crossover study on the efficacy of sildenafil, tadalafil, and vardenafil in the treatment of erectile dysfunction. J Sex Med 2009; 6(9):2547-60.

24. Hatzimouratidis K, Hatzichristou D. Phosphodiesterase type 5 inhibitors: the day after. Eur Urol 2007;51(1):75-88.

25. Bella AJ, Brant WO, Lue TF, et al. Non-arteritic anterior ischemic optic neuropathy (NAION) and phosphodiesterase type-5 inhibitors. Can J Urol 2006;13(5):3233-8.

26. Bella AJ, Deyoung LX, Al-Numi M, et al. Daily administration of phosphodiesterase type 5 inhibitors for urological and nonurological indications. Eur Urol 2007;52(4):990-1005.

27. Shindel AW. 2009 update on phosphodiesterase type 5 inhibitor therapy part 1: recent studies on routine dosing for penile rehabilitation, lower urinary tract symptoms, and other indications (CME). J Sex Med 2009;6(7):1794-808 [quiz: 1793, 1809-10].

28. Rubio-Aurioles E, Kim ED, Rosen RC, et al. Impact on erectile function and sexual quality of life of couples: a double-blind, randomized, placebo-controlled trial of tadalafil taken once daily. J Sex Med 2009;6(5):1314-23.

29. Shabsigh R, Kaufman JM, Steidle C, et al. Randomized study of testosterone gel as adjunctive therapy to sildenafil in hypogonadal men with erectile dysfunction who do not respond to sildenafil alone. J Urol 2004;172(2):658-63.

30. Heaton JP, Altwein JE. The role of apomorphine SL in the treatment of male erectile dysfunction. BJU Int 2001;88(Suppl 3):36-8.

31. Costabile RA, Mammen T, Hwang K. An overview and expert opinion on the use of alprostadil in the treatment of sexual dysfunction. Expert Opin Pharmacother 2008;9(8):1421-9.

32. Alexandre B, Lemaire A, Desvaux $P$, et al. Intracavernous injections of prostaglandin $\mathrm{E} 1$ for erectile dysfunction: patient satisfaction and quality of sex life on long-term treatment. J Sex Med 2007;4(2):426-31. 